\title{
High-frequency bistatic scattering by sub-bottom gas bubbles
}

\author{
Dezhang Chu \\ Department of Applied Ocean Physics and Engineering, Woods Hole Oceanographic Institution, \\ Woods Hole, Massachusetts 02543
}

Kevin L. Williams, Dajun Tang, and Darrell R. Jackson

Applied Physics Laboratory, University of Washington, Seattle, Washington 98105

(Received 5 March 1996; accepted for publication 24 March 1997)

\begin{abstract}
A previous study of high-frequency acoustic backscattering data collected at Eckernfoerde Bay, Germany revealed that scattering is mainly due to methane gas bubbles buried about a meter beneath the seafloor [Tang et al., J. Acoust. Soc. Am. 96, 2930-2936 (1994)]. A backscattering model was developed [Tang, Geo-Marine Lett. 16, 161-169 (1996)] where the gas bubbles were approximated by oblate spheroids. In this paper, a bistatic scattering model is proposed as an extension of the previously developed backscattering model. In this model, gas bubbles are again assumed to be oblate spheroids with varying aspect ratios and a single-scattering approximation is used. The model is compared to bistatic data acquired in Eckernfoerde Bay, Germany. In particular, the azimuthal dependence of the bistatic scattering strength predicted by the model is tested against experimental data and it is found that both the model and the bistatic scattering strength data exhibit a mild azimuthal dependence. Best agreement between model and data requires a 35\% reduction in areal bubble density relative to that used in the backscattering model/data comparison. Possible reasons for this are discussed including multiple scattering effects. (c) 1997 Acoustical Society of America.
\end{abstract} [S0001-4966(97)04307-5]

PACS numbers: 43.30.Ft, 43.30.Gv, 43.30.Ma [JHM]

\section{INTRODUCTION}

Sub-bottom contributions to acoustic scattering have received increased attention in recent years. It has been shown in a number of studies that sub-bottom inhomogeneities can dominate acoustic backscattering at frequencies as high as 40 $\mathrm{kHz} .{ }^{1,2}$ However, the previous investigations have been restricted to monostatic scattering (backscattering) in which the dependence of the scattering directivity pattern on receiving grazing and azimuthal angles cannot be examined. Although such a scattering directivity pattern can lead to further insight into the scattering physics, very few bistatic experiments of the type required to examine the scattering directivity pattern have been reported due to the engineering and operational difficulties involved in data acquisition.

Likewise, development of bistatic models is a more demanding task. Recently, Jackson ${ }^{3}$ developed a bistatic model including surface scattering from the rough water-sediment interface and volume scattering from the sub-bottom. The comparison of his model with experimental data shows a reasonable agreement. ${ }^{4}$ However, this model treats volume inhomogeneities via perturbation theory and, as such, does not address in a fundamental way environments in which scattering is due to high contrast scatterers such as bubbles. Just such an environment was encountered in Eckernfoerde Bay, Germany during the Coastal Benthic Boundary Layer Special Research Program (CBBL-SRP). Within the Eckernfoerde mud sediment, a layer consisting of many nonspherical methane gas bubbles was found at about $1 \mathrm{~m}$ below the smooth seafloor. ${ }^{5}$ These bubbles scatter sound far more efficiently than the water/seafloor interface and the mud above the bubble layer. ${ }^{1,2}$ In this paper, a two-layer bistatic scattering model is proposed to describe scattering by such a bubble layer and compared with data taken at the Eckernfoerde site. Section I outlines the theoretical development; Section II presents some results of numerical simulations, compares theory with experimental data, and ends with some discussion of possible multiple scattering effects. Conclusions are drawn in Sec. III.

\section{BISTATIC SCATTERING MODEL}

Bistatic scattering experiments employ configurations in which the transmitter and the receiver are at different positions. The commonly used monostatic scattering or backscattering geometry is a special case of the more general bistatic scattering geometry. Similar to backscattering strength, bistatic scattering strength can be defined as the decibel equivalent of the scattering cross section per unit area per unit solid angle ${ }^{3}$ and is, in general, a function of incident angles $\left(\theta_{i}, \phi_{i}\right)$ and scattering angles $\left(\theta_{s}, \phi_{s}\right)$, where $\theta$ and $\phi$ correspond to grazing and azimuth angles, respectively. When $\phi_{s}=\pi+\phi_{i}$, and $\theta_{s}=\theta_{i}$, bistatic scattering reduces to backscattering. Obviously, bistatic scattering measurements provide more information than backscattering measurements.

Previous backscattering studies at the Eckernfoerde Bay site showed that backscattering at $40 \mathrm{kHz}$ is due to a layer of methane gas bubbles located about $1 \mathrm{~m}$ below the seafloor, and the scattering by these bubbles has been successfully modeled by a backscattering model where the gas bubbles were approximated by nonresonant oblate spheroids. ${ }^{2}$ In addition to these backscattering data, bistatic scattering data were also collected with the same source and a mobile receiver at the same time and location. ${ }^{4}$ The purpose of this 
paper is to extend the backscattering model to the bistatic scenario.

Before introducing the model, we restate several assumptions made in the backscattering model that will be used also in the bistatic model. First of all, in Refs. 1 and 2, the surface scattering at $40 \mathrm{kHz}$ from the seafloor itself was found insignificant and could be ignored due to the weak acoustic impedance change across the seafloor/water interface.

Second, it is observed that the bubbles are concentrated in a layer distributed over a vertical distance of about $10 \mathrm{~cm}$. The differences between the grazing angles incident upon/ scattered from the top and bottom of the layer are small (less than $2^{\circ}$ for the geometry of the experiment). Since we consider single scattering only, it is reasonable to assume that all bubbles are on a surface with an equivalent bubble surface density obtained from integrating the volume density over depth. As a result, volume scattering is treated as quasisurface scattering and the propagation loss due to volume scattering is ignored. In contrast, the horizontal distribution of the bubbles is assumed to be homogeneous, i.e., a uniform probability density function (PDF).

Third, X-ray tomographic analysis of the core data ${ }^{6}$ shows that the shapes of the bubbles are nonspherical and their sizes vary from about $0.5 \mathrm{~mm}$ to less than $15 \mathrm{~mm}$ in volume-equivalent spherical radius. Smaller bubbles were not measured because of the limited resolution of the x-ray system. The bubbles are oriented as coins standing on their edges. Their broadside normals are, in general, parallel to the plane on which the bubbles are standing. For brevity, we call such a configuration horizontally oriented. To simplify our analysis and to objectively model bistatic scattering as in the backscattering model, we assume that resonant scattering is unimportant and can be neglected. In the present model, we assume that the bubbles can be properly modeled as horizontally oriented pressure-release oblate spheroids with various aspect ratios.

Finally, in the backscattering model, the effect of the multiple scattering among bubbles was not considered. Likewise, in the present bistatic scattering model multiple scattering will be ignored. This approximation will be revisited after model/data comparisons are presented.

\section{A. Geometry of the two-layer model}

A major issue of the two-layer model for a bistatic geometry is the determination of the ensonified region of the scattering layer. For a homogeneous medium, if the source signal is a short pulse, at any time greater than the minimum time required for the wave to travel from the source to the scattering layer and then to the receiver, the interception area on the scattering plane will be an elliptical ring whose width is proportional to the pulse length ${ }^{4}$ and can be determined analytically.

However, for the two-layer model, since there are two media (water column and sediment) with different sound speeds, the acoustic wave changes its propagation direction when it passes through the interface that divides the two media. The interception area at a given time instant is no longer an elliptical ring and its shape cannot be determined

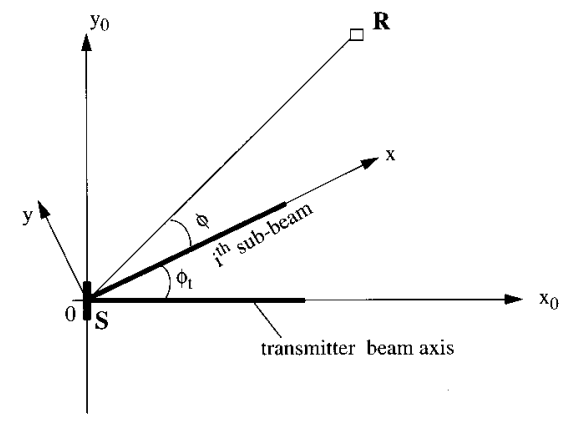

(a)

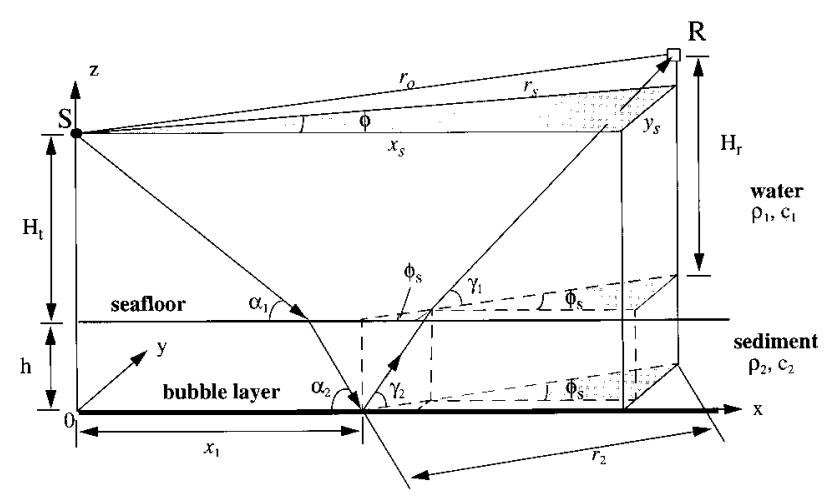

(b)

FIG. 1. Geometry of a bistatic scattering model. (a) Top view, where $\left(x_{0}, y_{0}\right)$ are original coordinates and $(x, y, z)$ are rotated coordinates. $S$ and $R$ stand for source and receiver, respectively. (b) Side view of the $i$ th subbeam.

analytically. To solve the problem numerically, we divide the transmit beam into a number of narrow, nonoverlapping sub-beams propagating in different directions, i.e., different azimuth angle $\phi_{t}$, and compute the scattering contribution due to each individual sub-beam. Since multiple scattering is not considered in the current model, the total scattered field can be obtained by simply summing up the contributions from all these sub-beams weighted by the transmitter and receiver beampatterns.

To compute the scattering due to each individual transmit sub-beam, a global coordinate system is chosen in such a way that the the acoustic source or transmitter is located at $x=0$, the $z$ axis is pointing up, and the bubble layer is at $z$ $=0$ as shown in Fig. 1, where Fig. 1(a) is the top view and Fig. 1(b) the side view. In Fig. 1(a) there are two coordinate systems: the original and rotated coordinate systems. Their $z$ axes coincide with each other pointing outward from the paper. The original coordinate system is chosen such that the $x_{0}$ axis coincides with the transmit beam axis, corresponding to zero azimuth angle. For a transmit sub-beam in an azimuthal direction $\phi_{t}$ (the angle between transmit beam axis and the sub-beam direction), the original coordinate system is rotated about the $z$ axis by an angle of $\phi_{t}$ to make the transmit azimuth angle zero in the rotated coordinate system $(x, y, z)$. The bistatic scattering geometry shown in Fig. 1(b) is the side view of the $i$ th transmit sub-beam. The transmitter is located at $\left(0,0, H_{t}+h\right)$ and the chosen sub-beam is in the 
$x-z$ plane, while the receiver position can be out of the $x-z$ plane and located at $\left(x_{s}, y_{s}, h+H_{r}\right)$, where $H_{t}$ and $H_{r}$ are distances from the seafloor to the transmitter and receiver correspondingly, and $h$ is the thickness of the sediment overlying the scatterers. From Fig. 1 the following geometric relations can be easily obtained:

$$
\begin{aligned}
& r_{s}=\sqrt{r_{0}^{2}-\left(H_{r}-H_{t}\right)^{2}}, \\
& x_{s}=r_{s} \cos \phi, \quad y_{s}=r_{s} \sin \phi, \\
& x_{1}=H_{t} \cot \alpha_{1}+h \cot \alpha_{2}, \\
& r_{2}=H_{r} \cot \gamma_{1}+h \cot \gamma_{2}, \\
& \phi_{s}=\sin ^{-1}\left(y_{s} / r_{2}\right), \quad x_{2}=r_{2} \cos \phi_{s},
\end{aligned}
$$

where $r_{0}$ is the distance between the transmitter and the receiver, $r_{s}$ the projection distance of $r_{0}$ to the $x-y$ plane, $\alpha_{1}$ and $\gamma_{1}$ are grazing angles at the seafloor, while $\alpha_{2}$ and $\gamma_{2}$ are grazing angles at the bubble layer. Note that $\alpha_{1}$ and $\alpha_{2}$ correspond to the transmitted wave while $\gamma_{1}$ and $\gamma_{2}$ correspond to the scattered wave. Using the above geometrical relations, we have

$$
\begin{aligned}
x_{s}= & x_{1}+x_{2} \\
= & H_{t} \cot \alpha_{1}+h \cot \alpha_{2}+\left(H_{r} \cot \gamma_{1}+h \cot \gamma_{2}\right) \\
& \times \cos \left[\sin ^{-1}\left(y_{s} /\left(H_{r} \cot \gamma_{1}+h \cot \gamma_{2}\right)\right)\right] .
\end{aligned}
$$

A constraint on the arrival time gives

$$
t_{s}=\frac{1}{c_{1}}\left(\frac{H_{t}}{\sin \alpha_{1}}+\frac{H_{r}}{\sin \gamma_{1}}\right)+\frac{h}{c_{2}}\left(\frac{1}{\sin \alpha_{2}}+\frac{1}{\sin \gamma_{2}}\right),
$$

where $c_{1}$ and $c_{2}$ are sound speeds of the water column and the sediment, respectively.

Grazing angles $\alpha_{1}, \alpha_{2}, \gamma_{1}$, and $\gamma_{2}$ in above equations are related by Snell's law:

$$
\begin{aligned}
& \cos \alpha_{2}=\frac{c_{2}}{c_{1}} \cos \alpha_{1}, \\
& \cos \gamma_{2}=\frac{c_{2}}{c_{1}} \cos \gamma_{1} .
\end{aligned}
$$

For a given time, there are only two independent unknowns in Eqs. (2) and (3): $\gamma_{1}$ and $\alpha_{1}$. These two simultaneous transcendental equations can be solved numerically. One way to solve the equations is to step through time intervals; $N$ discrete time steps, for example. It requires solving the transcendental equations $N$ times, which is not an efficient way.

An alternative way used in our modeling is to find all possible combinations of $\alpha_{1}$ and $\gamma_{1}$ that satisfy Eq. (2) first, then compute $t_{s}$ using Eq. (3) and the obtained $\alpha_{1}$ and $\gamma_{1}$ from Eq. (2), and finally sort the result according to an ascending time sequence $t_{s}$. This way we only need to solve the transcendental equations once.

It can be proved that when the receiver is in the same plane as the transmit sub-beam $\phi=0$, and when the scattering grazing angle $\gamma_{1}$ is equal to the transmit grazing angle $\alpha_{1}$, the travel time reaches its minimum, $t_{\min }$, and this scattering grazing angle $\gamma_{1}$ can be considered as the grazing angle in the specular direction, $\gamma_{\mathrm{sp}}$. At any other time $t_{s}$ $>t_{\text {min }}$, there are always two rays intercepting the gas-bubble layer, i.e., the $z=0$ plane: One corresponds to a grazing angle $\gamma_{1}$ larger than $\gamma_{\mathrm{sp}}$, and the other corresponds to a grazing angle smaller than $\gamma_{\mathrm{sp}}$. This bears some analogy to the case where only one homogeneous layer overlays the scattering layer and the ensonified area on the scattering layer is an elliptical ring. For any vertical plane containing the transmitting sub-beam and intercepting this scattering layer, there are always two intercepting points on the elliptical ring, corresponding to the two rays. For $\phi \neq 0$, there still exists a minimum travel time, $t_{\min }$, which corresponds to a single ray with the transmit and scattering grazing angles $\alpha_{1}$ and $\gamma_{1}$. Here $\alpha_{1}$ and $\gamma_{1}$ are not equal in general. At any time $t_{s}>t_{\min }$, there are still two rays with one ray having a scattering angle $\gamma_{1}$ greater than that corresponding to $t_{s}$ $=t_{\min }$, and the other smaller than $\gamma$. The total scattering contribution at time $t_{s}$ is the sum of these two rays.

\section{B. Scattering model}

As described in Refs. 1, 2, 5, and 6, the strong scattering layer consists of an aggregation of nonspherical gas bubbles which are modeled as (horizontally oriented) oblate spheroids with their major axis pointing randomly in directions orthogonal to the vertical. The model for bistatic scattering from these spheroids is an extension of the model developed by DiPerna and Stanton ${ }^{7}$ in which a conformal mapping method is applied. Since the bubbles have different orientations and aspect ratios, it is reasonable to assume the oblate spheroidal scatterer has an omnidirectional orientation distribution in the $x-y$ plane, i.e., bubbles are oriented uniformly over 0 to $2 \pi$. Obviously, for an oblate spheroid under such an assumption, the incident and azimuth angles with respect to the horizontal plane in the global coordinates or observation coordinates [rotated coordinates $(x, y, z)$ in Fig. 1(b)] are different from those with respect to the spheroid defined in the local coordinates (shown in Fig. A1). To perform a statistical average over orientation, coordinate transformations are needed to relate the local coordinates to global coordinates. The details of the transformations are given in the Appendix A. The average cross section over orientation as well as aspect ratio expressed in the global coordinates can be obtained by

$$
\begin{aligned}
\mathscr{S}_{s}\left(\gamma_{2}, \phi_{s} ; \alpha_{2}\right)= & \frac{2}{\pi} \int_{0}^{\pi / 2} \int_{-\infty}^{\infty} \sigma_{\epsilon}\left(\Theta_{s}, \Phi_{s i} ; \Theta_{i}\right) \\
& \times \rho(\epsilon) d \phi_{\text {rot }} d \epsilon,
\end{aligned}
$$

where $\sigma_{\epsilon}$ is the scattering cross section of an oblate spheroid with an aspect ratio $\epsilon, \Theta_{s}, \Phi_{s i}$, and $\Theta_{i}$ are given by Eqs. (A11)-(A15) in Appendix A, and $\rho(\epsilon)$ is the probability density function (PDF) of the aspect ratio $\epsilon$ given in the next section.

Using $\mathscr{S}_{s}\left(\gamma_{2}, \phi_{s} ; \alpha_{2}\right)$ together with $\alpha_{1}$ and $\gamma_{1}$ obtained numerically using the method described in the previous subsection, the received acoustic intensity corresponding to the transmit azimuthal angle $\phi_{t}$ at time $t$ for ray $i$ can be expressed as 


$$
\begin{aligned}
I_{s i}\left(\gamma_{2}, \phi_{s} ; \alpha_{2}, \phi_{t}\right)= & \frac{I_{0} e^{-\beta_{w} r_{w}} e^{-\beta_{s} r_{\mathrm{sed}}}}{r_{t}^{2} r_{\mathrm{scat}}^{2}} \mathscr{S}_{s}\left(\gamma_{2}, \phi_{s} ; \alpha_{2}\right) \\
& \times A \rho_{b} B_{t}\left(\theta_{t}, \phi_{t}\right) B_{r}\left(\theta_{r}, \phi_{r}\right) \\
& \times\left(T_{12} T_{21}\right),
\end{aligned}
$$

where $i=1,2$ corresponding to the two rays $\gamma_{1}<\gamma_{\mathrm{sp}}$ and $\gamma_{1}$ $>\gamma_{\mathrm{sp}}$, respectively,

$$
\begin{aligned}
& r_{t}^{2}=\left(\frac{H_{t}}{\tan \alpha_{1}}+\frac{h}{\tan \alpha_{2}}\right)\left(\frac{H_{t} \tan \alpha_{2}}{\sin ^{2} \alpha_{1}}+\frac{h \tan \alpha_{1}}{\sin ^{2} \alpha_{2}}\right), \\
& r_{\text {scat }}^{2}=\left(\frac{H_{r}}{\tan \gamma_{1}}+\frac{h}{\tan \gamma_{2}}\right)\left(\frac{H_{r} \tan \gamma_{2}}{\sin ^{2} \gamma_{1}}+\frac{h \tan \gamma_{1}}{\sin ^{2} \gamma_{2}}\right)
\end{aligned}
$$

are distances from the transmitter and the receiver to the scatterer, and

$$
\begin{aligned}
& r_{w}=\frac{H_{t}}{\sin \alpha_{1}}+\frac{H_{r}}{\sin \gamma_{1}}, \\
& r_{\text {sed }}=h\left(\frac{1}{\sin \alpha_{2}}+\frac{1}{\sin \gamma_{2}}\right)
\end{aligned}
$$

are travel distances of a ray in the water and in the sediment, respectively. Here, $\beta_{w}$ and $\beta_{s}$ are attenuation coefficients in water and sediment, respectively (neper/m); $T_{12}$ and $T_{21}$ are energy transmission coefficients from medium 1 to 2 and 2 to $1 ; \rho_{b}$ is the bubble surface density $\left(\right.$ no. $\left./ \mathrm{m}^{2}\right) ; B_{t}$ and $B_{r}$ are transmitter and receiver beampatterns with the arguments $\theta_{t}=\alpha_{1}-\theta_{\mathrm{td}}, \theta_{r}=\gamma_{1}-\theta_{\mathrm{rd}}$, and $\phi_{r}=\phi-\phi_{\mathrm{rd}}$, where $\theta_{\mathrm{td}}$ is the transmitter depression angle (mounting angle), and $\theta_{\mathrm{rd}}$ and $\phi_{\mathrm{rd}}$ are receiving depression and orientation angles accordingly. Here, $A$ is the ensonification area and can be determined numerically (Appendix B). Note that $\gamma_{2}, \phi_{s}, \alpha_{2}$, and $\phi_{t}$ are functions of time. The total received intensity at time $t, I_{s}(t)$ can then be obtain by integrating $I_{s i}$ given by Eq. (7) over $\phi_{t}$,

$$
I_{s}(t)=\int_{-\pi / 2}^{\pi / 2} \sum_{i=1}^{2} I_{s i}\left(\gamma_{2}, \phi_{s} ; \alpha_{2}, \phi_{t}\right) d \phi_{t},
$$

where we have summed up the contributions from the two rays corresponding to the transmit direction $\phi_{t}$. We ignored contributions for $\phi_{t}$ between $\pi / 2$ and $3 \pi / 2$ due to the strong attenuation of the transmitter beampattern (greater than 40 dB). In Eq. (10), at a given time, the angles $\gamma_{2}, \phi_{s}$, and $\alpha_{2}$ can be uniquely determined if a transmit azimuthal angle $\phi_{t}$ is specified. Therefore, a summation over $\phi_{t}$ in Eq. (10) also results in a summation over $\gamma_{2}, \phi_{s}$, and $\alpha_{2}$.

In general, contributing rays with a variety of incident and scattered angles intercept the bubble layer at any given time; therefore one cannot obtain the conventional scattering strength defined by a pair of incident and scattered angles. However, by taking advantage of the narrow beamwidths of both transmitter and receiver, it is possible to determine whether the scattered field is dominated by the contribution from a single direction. The average scattering strength (SS) per unit area per unit solid angle can be expressed as

$$
\operatorname{SS}\left(\gamma_{2}, \phi_{s} ; \alpha_{2}\right)=10 \log _{10}\left(\rho_{b} \mathscr{S}_{s}^{(d)}\left(\gamma_{2}, \phi_{s} ; \alpha_{2}\right)\right) \text {, }
$$

where the superscript $(d)$ denotes dominant scattering.

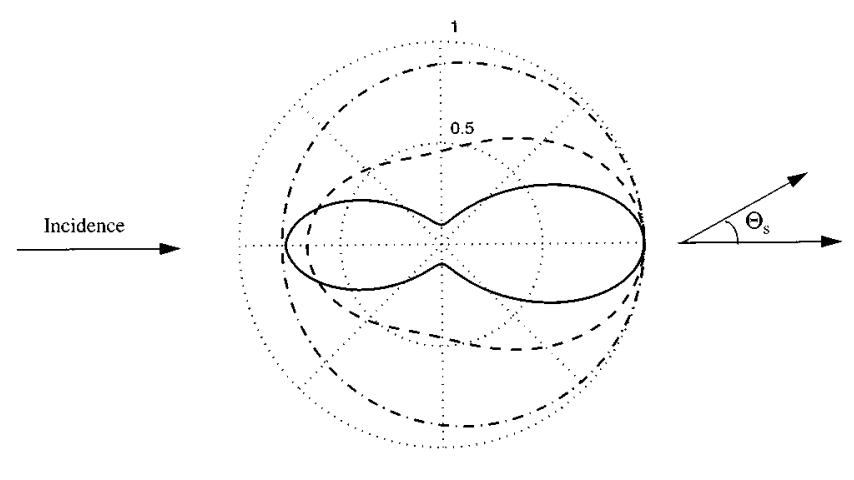

(a)

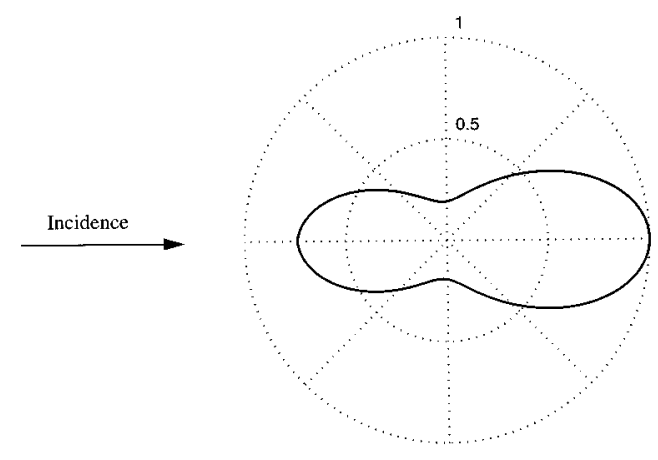

(b)

FIG. 2. Normalized bistatic scattering cross section of an oblate spheroid. In all computations, the frequency is $40 \mathrm{kHz}$, sound speed is $1425 \mathrm{~m} / \mathrm{s}$, and the semi-minor axis of the oblate spheroid is $1 \mathrm{~mm}$, resulting in $k a=0.18$. (a) Scattering directivity pattern of an oblate spheroid with an aspect ratio of 1 (dash-dotted), 5 (dashed), and 10 (solid); (b) normalized average scattering cross section over aspect ratio using an exponential PDF. In both (a) and (b) the incidence is along the broadside normal of the oblate spheroid, i.e., $\Theta_{i}$ $=0$ and the scattered angle $\Theta_{s}$ is the angle between the broadside normal and the scattered direction.

\section{RESULTS AND DISCUSSIONS}

\section{A. Numerical simulations}

At low frequencies, $k a \ll 1$, where $k$ is the wave number and $a$ is the characteristic geometric dimension of the object, scattering by a pressure-release sphere is almost omnidirectional. However it is directional for an oblate spheroid and approaches a dipolelike bistatic scattering pattern as aspect ratio increases. Figure 2(a) shows the normalized differential scattering cross section for a single oblate spheroid versus scattering angle $\Theta_{s}$ for a broadside incidence $\Theta_{i}=0$, where $\Theta_{i}$ and $\Theta_{s}$ are defined in a local coordinate system described in Appendix A. $\Theta_{i}$ represents the angle between the broadside normal and the incident direction, and $\Theta_{s}$ represents the angle between the broadside normal and scattering direction. Since the scattering is independent of azimuth angle, $\Phi_{s}$, only the scattering in an arbitrary azimuthal plane is illustrated. In the figure, $\Theta_{s}=0$ corresponds to forward scattering while $\Theta_{s}=180$ corresponds to backscattering. The three curves in Fig. 2(a) correspond to oblate spheroids with aspect ratio 1 (dashed-dotted), 5 (dashed), and 10 (solid), respectively. For the curve with aspect ratio of unity, i.e., a 


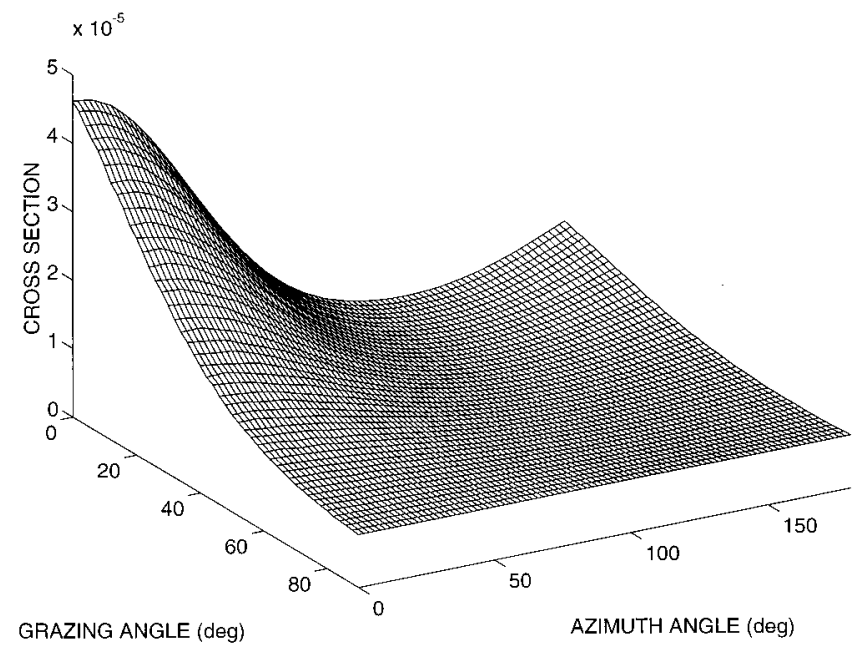

FIG. 3. Bistatic scattering cross section in the upper half-space for $\theta_{i}$ $=10^{\circ}$.

sphere, the variation in scattering cross section is less than $20 \%$, or $1 \mathrm{~dB}$, while for the curve with aspect ratio of 10 , the variation is greater than $80 \%$, or $7 \mathrm{~dB}$, and the cross section has a minimum at $\Theta_{s}=90^{\circ}$, showing a dipolelike scattering pattern. For the curve with aspect ratio 5 , the variation is within the two extremes.

For an aggregation of oblate spheroidal bubbles with various aspect ratios, an average scattering cross section can be obtained using an appropriate PDF. To perform the average, we assume that the semi-minor axis of the oblate spheroid is kept unchanged, while the semi-major axis changes according to the PDF. From the core data, ${ }^{8}$ it was observed that gas bubbles with smaller aspect ratios are more abundant than those with larger aspect ratios. A simple truncated exponential used in Ref. 2 is also used in our computation:

$$
\rho(\epsilon)=\left\{\begin{array}{l}
\frac{\beta}{e^{-\beta \epsilon_{\min }-e^{-\beta \epsilon_{\max }}} e^{-\beta \epsilon}, \quad \epsilon_{\min } \leqslant \epsilon \leqslant \epsilon_{\max }}, \\
0, \quad \text { otherwise }
\end{array}\right.
$$

with $\beta=0.25, \epsilon_{\min }=1$, and $\epsilon_{\max }=10$. Figure 2(b) illustrates the average scattering cross section normalized to unity and using such a truncated exponential PDF. It can be seen that, after averaging, the angular dependence has not been washed out entirely but is smoothed to some extent.

For a more general case, when the incidence is along an arbitrary direction (say $\theta_{i}$ as shown in Fig. A1) computations of the average bistatic cross section over orientations and aspect ratios given by Eq. (6) involve coordinate transformations described in Appendix A. Figure 3 shows a 3-D averaged bistatic scattering cross section in the upper half-space for $\theta_{i}=18^{\circ}$, where grazing and azimuth angles $\left(\theta_{s}, \phi_{s}\right)$ vary from $0-90^{\circ}$ and $0-180^{\circ}$, respectively. Similar to the azimuth independent case shown in Fig. 2, the variation of the scattering cross section is about $8 \mathrm{~dB}$. Again, it can be found that the maximum is in the forward direction while the minimum occurs at grazing angle $\theta_{s}=90^{\circ}$ when the azimuth angle $\phi_{s}$ is fixed or at azimuth angle $\phi_{s}=90^{\circ}$ when the grazing angle $\theta_{s}$ is fixed.
TABLE I. Parameters used the bistatic bottom scattering model.

\begin{tabular}{ll}
\hline \hline$c_{w}=1448 \mathrm{~m} / \mathrm{s}$ & sound speed in water \\
$c_{s}=1425 \mathrm{~m} / \mathrm{s}$ & sound speed in sediment \\
$\rho_{w}=1.0 \mathrm{~g} / \mathrm{cm}^{3}$ & water density \\
$\rho_{s}=1.1 \mathrm{~g} / \mathrm{cm}^{3}$ & sediment density \\
$\alpha_{w}=0 \mathrm{~dB} / \mathrm{m}$ & water attenuation coefficient \\
$\alpha_{s}=2.4 \mathrm{~dB} / \mathrm{m}$ & sediment attenuation coefficient \\
$\rho_{b}=8.8 \times 10^{3}\left(\right.$ no. $\left./ \mathrm{m}^{2}\right)$ & equivalent bubble surface density \\
\hline \hline
\end{tabular}

\section{B. Comparison with bistatic experimental data}

The $40-\mathrm{kHz}$ bistatic scattering data were collected at Eckernfoerde Bay, Germany in April, 1993. ${ }^{4}$ The Benthic Acoustic Measurement System (BAMS) served as the transmitter while the receiving array was suspended from a vessel that was moored at several locations in the vicinity of the source. The transmitter was mounted on a tripod $5 \mathrm{~m}$ above the seafloor, rotated $360^{\circ}$ in 72 steps with a step size of $5^{\circ}$, and transmitted one pulse at each step. The receiver was about $7.5 \mathrm{~m}$ above the seafloor and was within a 50-m range of the transmitter for the data shown here. The transmitted signal was a 2-ms linear frequency modulated signal, with its frequency swept from 39 to $41 \mathrm{kHz}$. Beampatterns of both transmitter and receiver are narrower in the horizontal plane (about $6.5^{\circ}$ beamwidth for both) and broader in the vertical plane $\left(14^{\circ}\right.$ and $37^{\circ}$ beamwidths for transmitter and receiver, respectively). Details of the system can be found in Ref. 4. Since the bistatic data were collected at the same location and the same time as the previously reported backscattering data, ${ }^{1,2}$ the parameters used in backscattering model were initially kept unchanged in the current bistatic scattering model. However, in the final model/data comparison to be shown here the bubble concentration, $\rho_{b}$ is reduced by about $35 \%$ (about $2 \mathrm{~dB}$ in scattering strength) to obtain the best fit. Possible reasons for this needed adjustment are discussed at the end of this section.

The parameters used in the modeling are listed in Table I, where all parameters are the same as those used in Refs. 1 and 2 except for bubble density discussed in the previous paragraph. The attenuation coefficient in the water column is set to zero since at $40 \mathrm{kHz}$ for a range within $50 \mathrm{~m}$, the attenuation is negligible.

In this study, a total of eight data sets have been analyzed. Each data set corresponds to one revolution $\left(360^{\circ}\right)$ of the transmitter ( 72 pings). Figure 4 shows the comparison of the reverberation level (RL) between the experimental data and the theoretical predictions for four pings from one data set, where the thinner lines are the experimental data and the thicker lines are the model predictions. These four pings are selected to represent the time series of the scattering from four different azimuthal directions and exhibit some distinct patterns. Since the transmission and reception systems used two independent internal clocks, the alignment of the data with the theoretical curves in time is adjusted primarily by aligning the first arrival of the data with the direct arrival predicted by the model based on the actual position and orientation information. For some pings, such adjustments could result in a misalignment between data and the theory, i.e., the major scattering structures (peaks and troughs) in the 

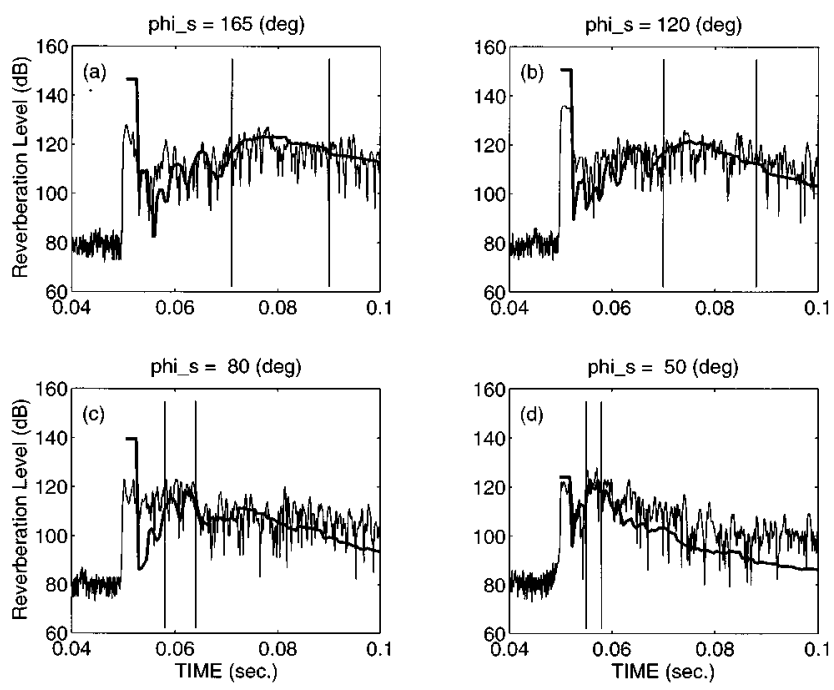

FIG. 4. Comparison of reverberation level between the bistatic scattering model predictions and the experimental data. The four time series are chosen from the same data set representing the bistatic scattering with four different azimuth angles as indicated in the figure. The vertical lines define the time windows within which the attenuation due to the beampattern (product of transmitting and receiving beampatterns) is less than $15 \mathrm{~dB}$, and the data are considered "reliable.",

RL time series can match much better if the RL curve is shifted a few ms. This could be attributed to the possible inaccuracy in determination of positions and orientations of the transducers.

From Fig. 4 it can be seen that the agreement between data and the model is reasonable in both scattering levels and patterns. When the scattering geometry approaches backscattering, i.e., the azimuthal angle between incident and scattering approaches $180^{\circ}$, the reverberation level (RL), after the initial direct arrival, gradually increases to its maximum, and then decreases slowly generating a broad peak as shown in (a) and (b). In contrast, when the scattering geometry changes from backward to forward, the peak becomes narrower and RL decreases faster than in the backward scattering case as shown in (c) and (d). Such a scattering characteristic is believed due to the narrow horizontal beamwidth (about $6.5^{\circ}$ ) but much broader vertical beamwidth of the transducers. For backscattering, the main lobes of the transmitter and receiver are overlapped throughout the entire data acquisition time interval associated with each ping, while for forward scattering the main lobes of the transmitter and the receiver intercept only over a much shorter time period, or may even not intercept at all (in which case the data are not analyzed further).

Another aspect of the comparison is the RL of the direct arrivals (the first large event in the time series). The differences between the prediction and the actual measurements are as large as $18 \mathrm{~dB}$. The error in RL caused by the errors in position data required in estimating the geometric spreading is insignificant, less than $0.4 \mathrm{~dB}$. However, the $\mathrm{RL}$ of the direct arrival is very sensitive to the orientation information since in many cases; the signal falls on the sharp edge (large gradient) of the main lobe or even within the sidelobes. Simulations reveal that $\mathrm{a} \pm 1^{\circ}$ error in orientation of the transducer or receiver can cause an error as large as $20 \mathrm{~dB}$ in estimating RL of the direct arrival. Consequently, this part of the data is not used in our analysis.

The analysis and discussions given above are also applicable to all other pings of the same data set and the other data sets. Generally speaking, the model fits the backward scattering data $\left(\phi_{s}>100^{\circ}\right)$ better than the forward scattering data. This could be explained, in part, by the beampattern effects. For the forward scattering, the main lobes of the transmit and receive beampatterns are partially overlapped, any is more sensitive to the accuracy of the orientation of the transducers than backward scattering.

Obviously, the total scattered field at any time results from the scattering from all directions. As noted earlier, it is not possible, in general, to obtain the relationship between the scattering cross section and the scattering angles as plotted in Fig. 3 since the scattering from a certain direction characterized by a particular sub-beam cannot be separated from the total scattered field. However, by taking advantage of the narrow horizontal beamwidths of the transducers, careful inspection makes it possible to determine whether the total scattered field is dominated by scattering from a certain direction. In our simulations, at any given time, when the ratio of the intensity in one dominant direction to the intensity in any other direction is greater than $15 \mathrm{~dB}$, we keep the data and designate that dominant direction as the primary scattering direction; otherwise we discard the data.

In addition, to avoid large errors due to the uncertain knowledge of sidelobes, a beampattern attenuation threshold is set to assure that all selected data fall in the main lobes. When the combined beampattern attenuation of transmitter and receiver is greater than a preset threshold $(15 \mathrm{~dB}$ used in the modeling), the data are considered "unreliable" and discarded. The selected data are bounded by the vertical lines, or the time windows shown in Fig. 4. The selected data are then averaged over this time window and converted to the average scattering strength of the bubble layer by the following equation:

$$
\begin{aligned}
\mathrm{SS}\left(\gamma_{2}, \phi_{s} ; \alpha_{2}\right)= & 10 \log _{10}\left\langle I_{\mathrm{sd}}\right\rangle-\mathrm{SL}+\alpha_{w} r_{w}+\alpha_{s} r_{\text {sed }} \\
& +20 \log _{10}\left(r_{t}\right)+20 \log _{10}\left(r_{\text {scat }}\right) \\
& -10 \log _{10} A-10 \log _{10} B_{t}\left(\theta_{t}, 0\right) \\
& -10 \log _{10} B_{r}\left(\theta_{r}, \phi_{r}\right) \\
& -10 \log _{10}\left(T_{12} T_{21}\right),
\end{aligned}
$$

where $\left\langle I_{\text {sd }}\right\rangle$ is received scattering data averaged over the selected time window, SL is the source level, and $\alpha_{w, s}$ $=\beta_{w, s} \log _{10} e$ is the attenuation coefficient in $\mathrm{dB} / \mathrm{m}$, and $A$ is, as before, the ensonification area. All physical properties of the water and sediments are given in Table I. All geometry parameters are those associated with the dominant scattering direction based on the actual position and orientation information. The resultant bistatic data points are plotted in Fig. 5 (plus signs) for the mean incident grazing angle $\left\langle\alpha_{2}\right\rangle=18^{\circ}$ with a deviation of $\pm 10^{\circ}$ and scattering grazing angle $\theta_{s}$ varying from $10^{\circ}$ to $20^{\circ}$, where the solid lines are theoretical curves computed from our bistatic model. The theoretical curves are averages over scattering grazing angle, 


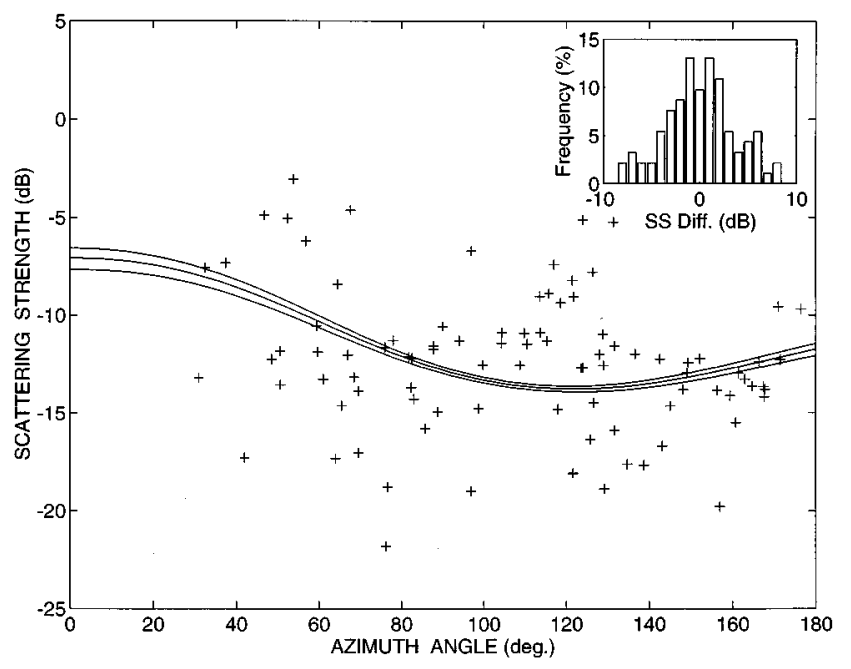

FIG. 5. Average bistatic scattering strength as a function of azimuth angle. The solid lines are the theoretical predictions given by Eq. (6) for incident grazing angle $\alpha_{2}=12^{\circ} \pm 10^{\circ}$ (uppermost), $18^{\circ} \pm 10^{\circ}$ (middle), and $24^{\circ}$ $\pm 10^{\circ}$ (bottom), respectively, where we have used an exponential PDF. The experimental data (plus) are obtained for incident grazing angle $\alpha_{2}=18^{\circ}$ $\pm 10^{\circ}$ and averaged over selected time window (bounded by vertical lines in Fig. 4). For both theoretical predictions and the data, the scattering grazing angle $\phi_{s}$ varies from $10^{\circ}$ to $20^{\circ}$. A superimposed plot at the upper right corner is the histogram of the difference of the scattering strength between theory $\left(18^{\circ} \pm 10^{\circ}\right)$ and the data.

$\theta_{s} 10^{\circ}-20^{\circ}$ and incident grazing angles, $\alpha_{2}$ of $12^{\circ} \pm 10^{\circ}$ (uppermost), $18^{\circ} \pm 10^{\circ}$ (middle), and $24^{\circ} \pm 10^{\circ}$ (bottom), respectively. The superimposed bar graph at the upper corner is the histogram of the difference between the experimental data and the theoretical curve of $\left\langle\alpha_{2}\right\rangle=18^{\circ}$ with a mean $\mu$ $=0.0 \mathrm{~dB}$ and a standard deviation $\sigma=3.7 \mathrm{~dB}$. Further analysis reveals that about $65 \%$ of all data points deviate less than $3 \mathrm{~dB}$ from the theoretical prediction and about $90 \%$ less than $6 \mathrm{~dB}$.

Figure 6 shows another comparison between the theory and data. The data are divided into 13 azimuth angle bands and processed by applying a median filter to the cross section

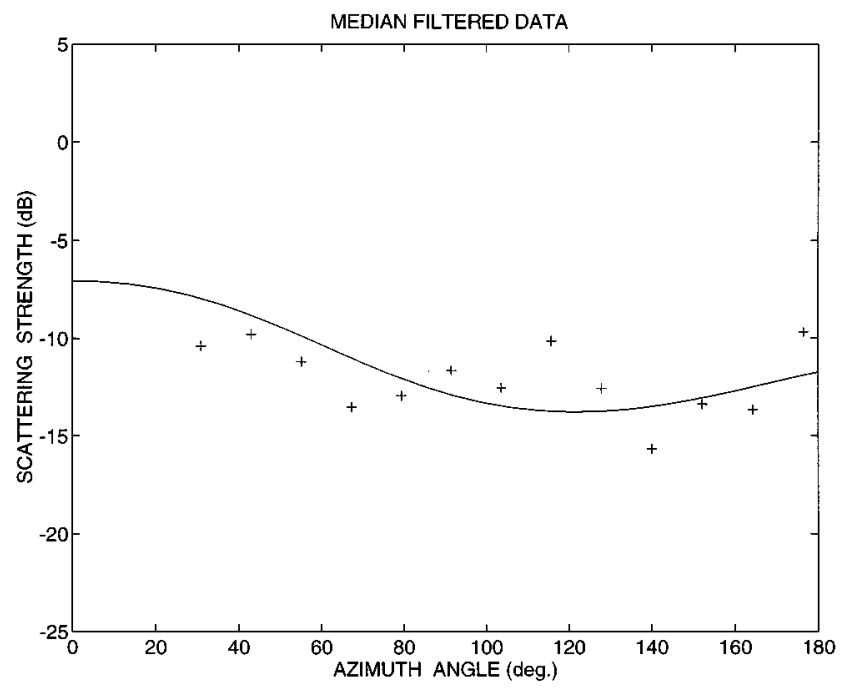

FIG. 6. Data presented in Fig. 5 are passed through a moving median filter over an azimuth angle window of $12^{\circ}$. within each angle band. The model prediction and the filtered data have similar angular dependence and fluctuations about the theoretical curve are less than $4 \mathrm{~dB}$.

\section{Forward scattering loss and multiple scattering effects}

The results presented here and in Ref. 2 demonstrate the capability of the model to quantitatively predict the measured acoustical scattering levels. One concern in the model/ data comparisons is that the bubble areal density needed to obtain best agreement in the present bistatic case is $35 \%$ lower than in the backscattering case. ${ }^{2}$ This is equivalent to a $2-\mathrm{dB}$ reduction in the bistatic scattering calculated via the model. At present we have three hypotheses as to why this might be so.

One hypothesis is that the calibrations of the sonar systems used in the backscattering and bistatic experiments have a differential error of $2 \mathrm{~dB}$. Error in any one calibration on the order of $1 \mathrm{~dB}$ cannot be ruled out.

Another possibility is that spatial variations of scattering in the region of the experiment are responsible for the difference in bubble density needed. Backscattering images in the vicinity of the tower show patchiness in the backscattering strength with variations on the order of $10 \mathrm{~dB}$ over scales of $10 \mathrm{~m}$ and a general lower level of scattering Northeast of the tower as compared to Southwest of the tower. ${ }^{9}$ Although the bistatic scattering and backscattering data are both acquired in the vicinity of the tower, a bias in levels could remain in the data due to the differing areas of the bottom sampled.

The third hypothesis is that the different bubble density needed in backscattering and bistatic scattering is due to multiple scattering and propagation loss. Enhanced backscattering is one possible ramification of multiple scattering $^{10}$ (an enhancement of $3 \mathrm{~dB}$ is possible), while propagation loss due to volume scattering (single or/and multiple scattering) as the acoustic wave propagates through the bubbly sediment results in a deduction in incident intensity. For brevity, we refer this type of propagation loss as forward scattering loss. A single scattering theory excluding forward scattering loss would lead to the need for a higher bubble density in the backscattering case as compared to the bistatic case.

There are at least two other indications of multiple scattering and forward scattering loss. The first is the downward looking sonar images taken (at $12 \mathrm{kHz}$ ) by Lambert et al. ${ }^{11}$ in the region of the experiment. In those images the bubble layer masks the signals below the layer, resulting in a dramatic reduction in the sound level below the layer.

Another (and most dramatic) indication comes from using the bistatic model to calculate the total scattered energy. The total scattered power from a unit ensonification area can be obtained by integrating the bistatic scattering cross section over all solid angles $\Omega$,

$$
P_{\mathrm{tot}}\left(\alpha_{2}\right)=I_{0} \int_{\Omega} \rho_{b} \mathscr{S}_{s}\left(\gamma_{2}, \phi_{s} ; \alpha_{2}\right) d \Omega
$$

where $I_{0}$ is the incident intensity at the scattering layer. 
If a surface scattering mechanism is assumed, the total incident power on a unit ensonified area (vertical energy flux) is $I_{0} \sin \left(\alpha_{2}\right)$. The ratios of the total scattered power $P_{\text {tot }}$ to the total incident power at the scattering layer are 10.5, 4.7, 2.1, and 0.8, for incident grazing angles $\alpha_{2}=5^{\circ}$, $10^{\circ}, 20^{\circ}$, and $40^{\circ}$, respectively. As incident angle $\alpha_{2}$ approaches zero, the ratio approaches infinity.

The fact that the ratios are greater than unity for incident angles below $34^{\circ}$ implies violation of the conservation of energy. Furthermore, even though the conservation of energy is not violated for the higher grazing angles, the high levels are still in violation of a single scattering assumption that relies on small scattering levels relative to the incident intensity. Further comments are included in Sec. III.

\section{CONCLUSIONS}

We have developed a two-layer, single scatter, bistatic model to simulate the scattering by nonspherical bubbles buried in an attenuating sediment. A bubble layer of a finite thickness is approximated by a bubble surface at a certain depth, i.e., all bubbles are on the surface. The bubbles are modeled as oblate spheroidal voids (pressure release).

When tested against data acquired in Eckernfoerde Bay, Germany, there is reasonable agreement if bubble density is reduced by $35 \%$ relative to that used in previous backscattering work for the same area. The average scattering strength exhibits a mild azimuthal dependence: Maximum scattering strength is reached in the forward direction and the minimum occurs when the transmitting and scattering directions are close to perpendicular with each other, while backscattering has a medium scattering level. The total fluctuation is about $6 \mathrm{~dB}$.

However, even though the single scattering theory seems quantitatively successful for the Eckernfoerde data in some aspects, further consideration of the amount of energy scattered indicates that it cannot be the whole story. Section II C implies that for the Eckernfoerde site multiple scattering and forward scattering loss must play a role at least for some range of grazing angles in order to avoid a violation of the conservation of energy. A more severe criterion is set by the data of Lambert et al. ${ }^{11}$ that indicates at least qualitatively that the two scattering mechanisms are probably important even at normal incidence. This implies that multiple scattering and forward scattering loss are in effect being treated phenomenologically in the present single scattering theory via reduction in bubble density, whereas what is probably happening is that the deeper bubbles in the bubble layer are seeing a reduced field. Consequently predictions for scattering back into the water column are consistent with those measured but predictions of the amount of energy in the sediment below the bubble layer violate both conservation of energy and the more qualitative results of Lambert et al. ${ }^{11}$ Therefore, further modeling of the Eckernfoerde site that incorporates multiple scattering and forward scattering loss is desired.

This motivation for incorporation of multiple scattering and forward scattering loss is actually more general. Regardless of bubble densities, shallow incidence angles may lead to multiscattering effects. In a qualitative sense, this is be- cause as the incidence angle gets shallower the pathlength through the bubble layer gets longer and the possibility of scattering from multiple bubbles and the forward scattering loss increases.

\section{ACKNOWLEDGMENTS}

This work is supported by the Coastal Benthic Boundary Layer Special Research Program, Office of Navel Research Grant No. N00014-95-1-G904. This is the Woods Hole Oceanographic Institution Contribution No. 9421.

\section{APPENDIX A}

To obtain an average bistatic scattering model of an oblate spheroid over orientation and aspect ratio, it is necessary to express the required spherical angles $(\Theta, \Phi)$ in local coordinates $(X, Y, Z)$ (modeling coordinates) in terms of $(\theta, \phi)$ in the global coordinates $(x, y, z)$ (observation coordinates). The local coordinate system is chosen in such a way that the $Z$ axis coincides with the $z$ axis, and the $X$ axis coincides with the broadside unit normal of the oblate spheroid. As shown in Fig. A1, the incident and scattered wave vector can be expressed in global coordinates as

$$
\begin{aligned}
& \hat{k}_{i}=\left(\cos \theta_{i}, 0,-\sin \theta_{i}\right), \\
& \hat{k}_{s}=\left(\cos \theta_{s} \cos \phi_{s}, \cos \theta_{s} \sin \phi_{s}, \sin \theta_{s}\right) .
\end{aligned}
$$

The broadside unit normal of the oblate spheroid, $\hat{n}$, lies in the $x-y$ plane and rotates about the $z$ axis by an angle of $\phi_{\text {rot }}$, representing different orientation of the oblate spheroid. To express the local coordinates $(X, Y, Z)$ in terms of the global coordinates $(x, y, z)$, we utilize the following coordinate transformations:

$$
\begin{aligned}
& \hat{k}_{x}=l_{1} \hat{K}_{x}+l_{2} \hat{K}_{y}+l_{3} \hat{K}_{z}, \\
& \hat{k}_{y}=m_{1} \hat{K}_{x}+m_{2} \hat{K}_{y}+m_{3} \hat{K}_{z}, \\
& \hat{k}_{z}=n_{1} \hat{K}_{x}+n_{2} \hat{K}_{y}+n_{3} \hat{K}_{z},
\end{aligned}
$$

where, again, upper case letters denote vector components in the local coordinate system. The direction cosines of the three rotated axes can be easily obtained

$$
\left(l_{1}, m_{1}, n_{1}\right)=\left(\cos \phi_{\text {rot }}, \sin \phi_{\text {rot }}, 0\right),
$$

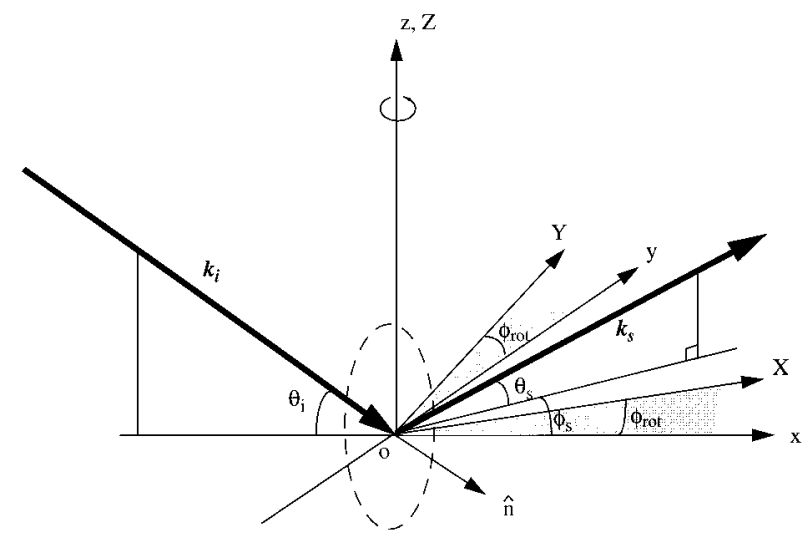

FIG. A1. Geometry of global to local coordinates transformation. 


$$
\begin{aligned}
& \left(l_{2}, m_{2}, n_{2}\right)=\left(-\sin \phi_{\mathrm{rot}}, \cos \phi_{\mathrm{rot}}, 0\right), \\
& \left(l_{3}, m_{3}, n_{3}\right)=(0,0,1),
\end{aligned}
$$

where $\phi_{\text {rot }}$ is the rotation angle. Using these equations, we can express the unit vectors of incident and scattered waves in the local coordinates in terms of the spherical angles defined in global coordinates $(x, y, z)$ as

$$
\begin{aligned}
\hat{K}_{i}= & \left(\cos \theta_{i} \cos \phi_{\text {rot }},-\cos \theta_{i} \sin \phi_{\text {rot }},-\sin \theta_{i}\right), \\
\hat{K}_{s}= & \left(\cos \theta_{s} \cos \left(\phi_{s}-\phi_{\text {rot }}\right), \cos \theta_{s}\right. \\
& \left.\times \sin \left(\phi_{s}-\phi_{\text {rot }}\right), \sin \theta_{s}\right) .
\end{aligned}
$$

Therefore, the two spherical angles $(\Theta, \Phi)$ of the incident and scattered waves in local coordinates $(X, Y, Z)$ are

$$
\begin{aligned}
& \cos \Theta_{i}=\hat{K}_{i x}=\cos \theta_{i} \cos \phi_{\text {rot }}, \\
& \cos \Theta_{s}=\hat{K}_{s x}=\cos \theta_{s} \cos \left(\phi_{s}-\phi_{\text {rot }}\right), \\
& \tan \Phi_{i}=\frac{\hat{K}_{i z}}{\hat{K}_{i y}}=\tan \theta_{i} \csc \phi_{\text {rot }}, \\
& \tan \Phi_{s}=\frac{\hat{K}_{s z}}{\hat{K}_{s y}}=\tan \theta_{s} \csc \left(\phi_{s}-\phi_{\text {rot }}\right) .
\end{aligned}
$$

It is convenient to choose a coordinate system in which the incident azimuth angle $\Phi_{i}$ is zero. To do so, we simply rotate the $Y$ - $Z$ plane about $X$ axis by $\Phi_{i}$ because of the symmetry of an oblate spheroid, the new scattering azimuth angle is found to be

$$
\Phi_{s i}=\Phi_{s}-\Phi_{i} .
$$

\section{APPENDIX B}

The ensonified area is a function of the difference of the incident and scattering angles, pulse length, sound speeds of water, and sediment. For a homogeneous medium, the ensonified area at any time $t>t_{\min }$, where $t_{\min }$ is the earliest scattering arrival from the scattering layer, can be described as an elliptical ring, the outer and inner ellipses correspond two curves of equal arrival times at $t-\tau$ and $t$, respectively. ${ }^{4}$ However, for a two-layer scattering geometry, since refractions across the two-layer interface greatly complicate the geometry, the ensonified area, in general, cannot be determined analytically and can only be achieved numerically. A plan view of the ensonified area for a bistatic scattering geometry is depicted schematically in Fig. B1. In the figure, $\mathbf{k}_{\mathbf{i}}$ and $\mathbf{k}_{\mathrm{s}}$ are incident and scattering wave vectors, $\overline{a_{1} a_{2}}$ and $\overline{b_{1} b_{2}}$ are wavefronts of the transmitted and scattered waves, respectively. $\overline{a_{1} a_{2}}=x_{1} \Phi_{t b}$, where $x_{1}$ is transmit horizontal distances given by Eq. (1), $\Phi_{t b}$ is the sub-beam beamwidth of the transmitter (see Sec. I A). Interception point $O$ corresponds to the earliest arrival. The difference of arrival time between $O$ and any point in the plane (scattering or bubble layer) is

$$
\Delta_{t}=\frac{1}{c_{2}}\left(\overline{P R} / \cos \alpha_{2}+\overline{R Q} / \cos \gamma_{2}\right),
$$
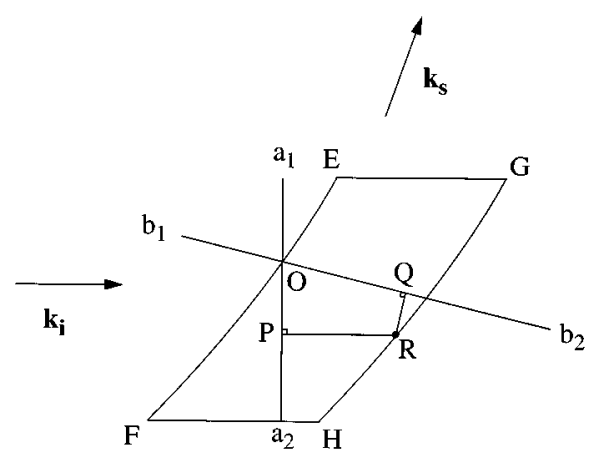

FIG. B1. Schematic description of the ensonified area for a bistatic scattering geometry.

where incident and scattering grazing angles $\alpha_{2}$ and $\gamma_{2}$ can be obtained numerically. Note that $\overline{P R}$ is positive when point $R$ is on the right of $\overline{a_{1} a_{2}}$ and negative on the left. Positive value corresponds to a time delay while negative value corresponds to a time advance. Similarly, $\overline{Q R}$ is positive when $R$ is below $\overline{b_{1} b_{2}}$ and negative when it is above $\overline{b_{1} b_{2}}$.

Two arcs $\widehat{E F}$ and $\widehat{G H}$ represent two equal arrival time lines for $t-\tau$ and $t$, respectively. They can be obtained by setting $\Delta_{t}=0$ and $\Delta_{t}=\tau$ in Eq. (B1), where $\tau$ is the pulse length. The ensonified area at time $t$ for the sub-beam considered is bounded by the two equal time lines and the two segments $\overline{E G}$ and $\overline{F H}$.

${ }^{1}$ D. Tang, G. Jin, D. R. Jackson, and K. L. Williams, “Analysis of highfrequency bottom and subbottom backscattering for two distinct shallow water environments," J. Acoust. Soc. Am. 96, 2930-2936 (1994).

${ }^{2}$ D. Tang, "Modeling high-frequency acoustic backscattering from gas voids," Geomarine Lett. 16, 261-265 (1996).

${ }^{3}$ D. R. Jackson, "Models for scattering from the seabed," Proc. Inst. Acoust. 16, 161-169 (1994).

${ }^{4}$ K. L. Williams and D. R. Jackson, "Monostatic and bistatic bottom scattering: Recent modeling and experiments,' Proceedings of OCEANS '94, Vol. II, 255-260 (1994).

${ }^{5}$ A. L. Anderson and F. Abegg, "Measurement of gas bubble concentration at Eckernfoerde Bay, Germany," EOS Trans. Am. Geophys. Union 75, 159 (1994).

${ }^{6}$ F. Abegg, A. Anderson, L. Buzi, A. P. Lyons, and T. H. Orsi, "Free methane concentration and bubble characteristics in Eckernfoerde Bay, Germany," in Proceedings of the Gassy Mud Workshop, edited by T. F. Wever held at the FWG, Kiel, Germany, 1994, pp. 84-89.

${ }^{7}$ D. T. DiPerna and T. K. Stanton, "Sound scattering by cylinders of nonspherical cross section: A conformal mapping approach,' J. Acoust. Soc. Am. 96, 3064-3079 (1994). Details on the extension of cylinders to spheroids can be obtained through private communication with D. T. DiPerna.

${ }^{8}$ T. H. Orsi, A. L. Anderson, and A. P. Lyons, "X-ray tomographic analysis of sediment macrostructure and physical property variability in Eckernfoerde Bay sediments, Western Baltic Sea,' Geomarine Lett. 16, 232239 (1996).

${ }^{9}$ D. R. Jackson, K. L. Williams, and K. B. Briggs, "High-frequency acoustic observations of benthic spatial and temporal variability," Geomarine Lett. 16, 212-218 (1996).

${ }^{10}$ A. Ishimaru, "Backscattering enhancement: From radar cross-sections to electron and light localizations to rough surface scattering,"' IEEE Trans. Antennas Propag. 33, 7-11 (1991).

${ }^{11}$ D. N. Lambert, D. J. Walter, and J. A. Hawkins, “Delineation of shallow, subbottom gas concentrations using a narrow beam, high frequency acoustic system," Proceedings of the workshop on Modeling Methane-Rich sediments of Eckernfoerde Bay, Eckernfoerde, 26-30 June 1995. 